\title{
QTAIM-based local potential energy model: applications and limitations to quantify the binding energy of intra/intermolecular interactions.
}

Caio L. Firme

Federal University of Rio Grande do Norte (UFRN), Institute of Chemistry

Av. Senador Salgado Filho, 3000, Central Campus, Natal, Brazil, CEP:59078-970.

Phone number: +55(84)33422323. Ext:144

e-mail: firme.caio@gmail.com or caiofirme@quimica.ufrn.br

Keywords: QTAIM; Local potential energy; LPE; energy decomposition analysis; supramolecular energy. binding energy; intermolecular interactions; non-conventional hydrogen bond.

\begin{abstract}
In previous work, we developed the local potential energy model, LPE, based on the electrostatic force and QTAIM topological data to quantify classical hydrogen bond energies. In this work, we extended the investigation to other inter/intramolecular interactions (non-conventional hydrogen bonds and others). The LPE presented high precision and linearity with supramolecular binding energy, when excluding interactions of an ion with $\pi$-bonded groups or polar molecule. The energy decomposition analysis from SAPT-DFT and LMOEDA showed that dispersion and electrostatic components are important to LPE, while polarization component impairs it. The LPE cannot be used for complexes with predominant polarization component.
\end{abstract}

\section{INTRODUCTION}

The inter/intramolecular interactions comprise a set of interatomic interactions where chemical bonds (such as ionic, covalent, metallic or dative bonds) are not included. The most important is the hydrogen bond which can be classified as classical or non-conventional. The classical hydrogen bond is made of a hydrogen atom bounded to two high electronegative atoms (N, O, S or halogen). For unsymmetrical hydrogen bonds, there is one strong covalent bond and a weaker interaction, represented by Dn-H---Ac, where Dn and Ac means (hydrogen) donor and (hydrogen) acceptor atoms, respectively [1,2]. For symmetric hydrogen bonds, two atoms of the same chemical element (e.g, halogen atoms) are bonded to the hydrogen atom with the same interatomic distance [3,4]. The classical hydrogen bonds are very important for biological systems including the protein synthesis and structure, the DNA structure, the enzyme-substrate interaction, and so on [5-7].

In addition, the classical hydrogen bonds have an important component of the electrostatic force [8], although they also have a degree of covalency or shared charge density between acceptor and hydrogen atoms [9]. In fact, according to the energy decomposition analysis [10-12], any interatomic interaction has the contribution of the following components: (1) energy from electrostatic force, (2) energy from polarization or induction, (3) energy from dispersion force and (4) energy from exchange interaction. Nonetheless, the unique fundamental force in all types of chemical bonds and inter/intramolecular 
interactions (including dispersion forces) is the electromagnetic force [13], while the exchange interaction is a quantum mechanical effect.

The quantum theory of atoms in molecules, QTAIM, has several applications for the study of the hydrogen bonds: (1) it is an important tool to characterize a hydrogen bond from topological data [9]; (2) it has topological descriptors for predicting the hydrogen bond [14,15]; and (3) it has parameters to measure the strength of the hydrogen bond [16-19].

The non-conventional hydrogen bond comprises $\mathrm{C}-\mathrm{H}$ bond as hydrogen donor or carbanions/carbenes/silylenes as hydrogen acceptor or $\pi$-bonded functional groups as hydrogen acceptor[20]. For example, in the $\pi$-complexes of the hydrochlorination of alkenes there is a non-conventional hydrogen bond between hydrogen (from hydrogen chloride) and $\pi$-bond of alkenes[21].

Besides classical and non-conventional hydrogen bonds, there are other interatomic interactions such as $\pi$-stacking, halogen bond, hydrogen-hydrogen bond, induced dipole-dipole interaction, ion-dipole interaction, and so on. All these interaction can be analyzed by QTAIM [22]. The $\pi$-stacking is an interaction between two displaced benzenoid rings. The $\pi$-stacking can be observed in the cyclophanes compounds containing alkyl-bridged mono/multilayered phenyl rings [23]. The hydrogen-hydrogen bond is an interaction between two nearly-zero charged hydrogen atoms bonded to carbon atoms (C-H---H-C) observed in alkane and alkene complexes [24,25]. The halogen bond is an interatomic interaction involving halogen atoms of distinguished molecules or halogen atom with another nucleophilic site [26].

In our previous work[27], we have developed the local potential energy model, LPE, a new method to quantify the binding energy of inter/intramolecular interaction using QTAIM and the electrostatic force model. We have shown that the LPE can be used to quantify the binding energy of classical hydrogen bonds[27] with relatively high precision. In this work, we extended the analysis of LPE to other interatomic interactions including the hydrogen bond involving formal charge in the acceptor (not investigated in the previous work).

\section{COMPUTATIONAL DETAILS}

The geometry of all studied molecules were optimized using the Berny algorithm [28] and direct inversion in interactive subspace method[29,30] from Gaussian 09 software [31]. Frequency calculation confirmed that all studied molecules, except for the transition state of 2-nitrophenol, are minima in the potential energy surface. The level of theory used for all optimization and frequency calculations was $\omega B 97 X-D[32] /$ aug-cc-pVTZ[33,34]. This level of theory had the best results for the analysis of the distance dependence of the hydrogen bond energy from a set of DFT functionals and basis sets[35]. The wave function for further QTAIM calculations was also obtained from the $\omega$ B97X-D[32]/aug-cc-pVTZ level of theory. The optimized geometries of all dimers and complexes were also used as input for LMOEDA and SAPT-DFT methods. The energy decomposition analysis based on SAPT/DFT[10,11] was obtained from PSI4[36] software using the $\omega$ B97X-D[32]/aug-cc-pVTZ level of theory. The GAMESS-US package [37] was used to obtain the energy decomposition analysis based on LMOEDA[12] with M06-2X [38]/ 6-311G++(d,p) level of theory. All QTAIM data for the local potential energy were obtained from AIM2000 software [39]. The $\omega$ B97X-D showed the best results in the assessment of performance of DFT and DFT-D functionals for hydrogen bond interactions [35]. 


\section{LOCAL POTENTIAL ENERGY, LPE}

The local potential energy, LPE, is based on the Coulomb's law for two charged particles. The unique fundamental force in chemical bonds and inter/intramolecular interactions is the electromagnetic force where the most relevant is the electrostatic force [13].

The general equation (in SI units) of the electrostatic force and corresponding potential energy in a hydrogen-like atom is given in Eq.(1) and (2), respectively.

$$
\begin{gathered}
F_{(H-l i k e)}=K \frac{Z_{e f f} e^{2}}{r^{2}} \\
V_{(H-l i k e)}=K \frac{Z_{e f f} e^{2}}{r}
\end{gathered}
$$

Where $\mathrm{K}\left(\mathrm{K}=9 \times 10^{9} \mathrm{~N} \mathrm{~m}^{2} \mathrm{C}^{-2}\right)$ is the Coulomb constant, $Z_{\mathrm{eff}}(\mathrm{e})$ is the effective charge of the hydrogen-like nucleus, $\mathrm{e}$ is the electron charge $\left(\mathrm{e}=-1.602 \times 10^{-9} \mathrm{C}\right)$, and $\mathrm{r}$ is the distance vector. In atomic units, $\mathrm{K}=1, \mathrm{e}=1$ and $r$ is given in Bohr unit. The corresponding equations for Eq. (1) and (2) in atomic units are given by Eq. 3 and 4.

$$
\begin{gathered}
F_{(H-l i k e)}=\frac{Z_{e f f}}{r^{2}} \\
V_{(H-l i k e)}=\frac{Z_{e f f}}{r}
\end{gathered}
$$

In the local potential energy model, QTAIM descriptors are used in the Coulomb's law to quantify the binding energy of the intra/intermolecular interactions of dimers or complexes.

According to the QTAIM, in each bounded system in its equilibrium geometry, there is a set of paired bond paths (or virial paths) uniting atomic basins (known as nuclear attractor critical point, NA) and the charge density bond critical point (BCP). The total number of critical points follows the Poincaré-Hopf relation, $n-b+r-c=1$, where $n$ is the number of nuclear attractors [a $(3,-3)$ critical point of the charge density], $b$ is the number of bond critical points of the charge density $[a(3,-1)$ critical point], $r$ is the number of ring critical points of the charge density $[\mathrm{a}(3,+1)$ critical point $]$ and $\mathrm{c}$ is the number of cage critical points of the charge density $[a(3,+3)$ critical point]. A $(3,-3)$ critical point is a maximum point at three directions of the charge density and a $(3,-1)$ critical point is a maximum point in two directions. The set of bond paths and charge density critical points is known as molecular graph. In addition, a requisite for the molecular graph is to be mirrored by a virial graph - a corresponding set of virial paths and potential energy density critical points.

In our book [22], there is an alternative presentation of the most important intermolecular interactions based on QTAIM and electrostatic force. We have used the electrostatic force model qualitatively or semi-quantitatively to rationalize our DFT-D supramolecular binding energy of several dimers or complexes as representative cases of the most important intermolecular interactions. Then, our book is a complementary view of this present work which also gave birth to the LPE model. 
The local potential energy model uses the potential energy equation of the electrostatic force model applying the QTAIM descriptors (Eq.5) on it. The LPE equation is an average of the sum of the potential energy from two electrostatic interactions: $\rho_{\mathrm{bcp}}$ interacting with $\mathrm{Z}_{\mathrm{eff}(\mathrm{H})}$ and $\rho_{\mathrm{bcp}}$ interacting with $\mathrm{Z}_{\mathrm{eff}(\mathrm{Ac}) \text {. }}$

$$
L P E_{\text {interaction }}=\frac{1}{2}\left(\frac{\rho_{b c p} Z_{e f f(I)}}{r_{I-b c p}}+\frac{\rho_{b c p} Z_{e f f(I I)}}{r_{I I-b c p}}\right)
$$

Where $\rho_{b c p}$ is the charge density of the bond critical point, $\mathrm{r}_{\mathrm{I}-\mathrm{bcp}}$ and $\mathrm{r}_{\mathrm{II}-\mathrm{bcp}}$ are the distance of the bond path from the bond critical point to the interacting atoms I and II (see Fig.1), respectively, in Bohr unit, and $\mathrm{Z}_{\mathrm{eff}}$ is the effective atomic number given by Eq.6, based on QTAIM descriptors as well:

$$
Z_{e f f(i)}=Z_{i}+(-1) \cdot q_{i}-L I_{i}
$$

Where $\mathrm{Z}_{\mathrm{i}}, \mathrm{q}_{\mathrm{i}}$ and $\mathrm{LI}_{\mathrm{i}}$ are the atomic number, QTAIM atomic charge and localization index of the ith atom, respectively. In the last equation, we assume LI as the screening constant.

The localization index of the ith atomic basin, $\mathrm{LI}_{\mathrm{i}}$, is the total number of electrons localized in this atomic basin, given by the double integration over the ith atomic basin, $\Omega$, of the exchange density, $\mathrm{P}_{\mathrm{XC}}$ (Eq.7), which can alternatively be approximated to the sum of squares of overlap integrals, $S$ [49].

$$
L I=-\iint_{\Omega} P_{X C}\left(r_{1}, r_{2}\right) d r_{1} d r_{2} \simeq-\sum_{i, j}^{N \alpha} S_{i, j}^{2}(\Omega)
$$

\section{RESULTS AND DISCUSSION}

We have chosen molecular systems which encompass a wide range of intermolecular interactions. The helium diatomic (1) has an induced (atomic) dipole-induced (atomic) dipole interaction. The argonium-hydrogen chloride (2) has an (atomic) induced dipole-dipole interaction. Both helium and argonium have an induced atomic dipole in the presence of other noble gas or polar molecule. For example, in argonium atom, its QTAIM atomic dipole in $\mathbf{2}$ is $0.0042 \mathrm{au}$. and $0.0003 \mathrm{au}$. isolated. The chloromethane dimer (3) has a non-conventional hydrogen bond with $\mathrm{C}-\mathrm{H}$ as hydrogen donor and chlorine as acceptor. The tetrafluoromethane dimer (4) has a halogen bond. The methyl ether dimer (5) has a non-conventional hydrogen bond with $\mathrm{C}-\mathrm{H}$ as hydrogen donor and oxygen as acceptor. The ethyne-water complex interacting from $\mathrm{CH}---\mathrm{O}$ (6) has a non-conventional hydrogen bond with $\mathrm{C} \equiv \mathrm{C}-\mathrm{H}$ as hydrogen donor and oxygen as acceptor[41]. The propane-dimer (7) has a hydrogen-hydrogen bond(ing). The formaldehyde-chlorine (8) has a non-conventional hydrogen bond with $\mathrm{O}=\mathrm{C}-\mathrm{H}$ as hydrogen donor and chlorine as acceptor. Important to note in Fig. 1 that there are secondary interactions in dimers of $\mathbf{5}$ and $\mathbf{8}$ (hydrogen-hydrogen bond and halogen bond, respectively). The benzene-dimer (9) has a $\pi$ stacking interaction. The 2-nitrophenol (10) has an intramolecular hydrogen bond with partial formal charge in the acceptor. As to the complexes benzene-nitrosonium ion (11), propene-hydrogen chloride (12) and ethyne-hydrogen fluoride (13), there is a non-conventional hydrogen bond with $\pi$-bond system as hydrogen acceptor from an aromatic ring, $\mathrm{CC}$ double bond and CC triple bond, respectively. The complex $\mathbf{1 1}$ is an example of $\pi$ complex from electrophilic aromatic substitution and the complexes $\mathbf{1 2}$ and $\mathbf{1 3}$ are examples of $\pi$ complexes from electrophilic addition to alkenes/alkynes. Finally, the chloromethane-sodium chloride complex (14) has ion-dipole interaction. 
The Fig. 1 shows the molecular graph of the optimized geometries from dimers/complexes $\mathbf{1}$ to $\mathbf{9}$. It also indicates the pair of bond paths associated with the intermolecular interaction involving the interacting atoms I and II, where $r$ means the distance (in Bohr) displayed in Table 1. The Fig.1 also depicts all distinguished LPEs in the same complex, represented by LPE1, LPE2, so on. When the bond paths of the interatomic interactions are equivalent in a complex (i.e., they have the same charge density at the bond critical point and bond path length), there are repeated designations of the LPE. This is the case for the molecular graphs of dimer $\mathbf{4}$ where there are two repeated LPE's, LPE2 and LPE3, and dimer 5, where there is one repeated LPE, LPE1. In the complex 6, it is referred as ethyne-water(CH---O) to distinguish it from the other possible interaction in this system (a non-conventional hydrogen bond where $\pi$ bond is the acceptor).
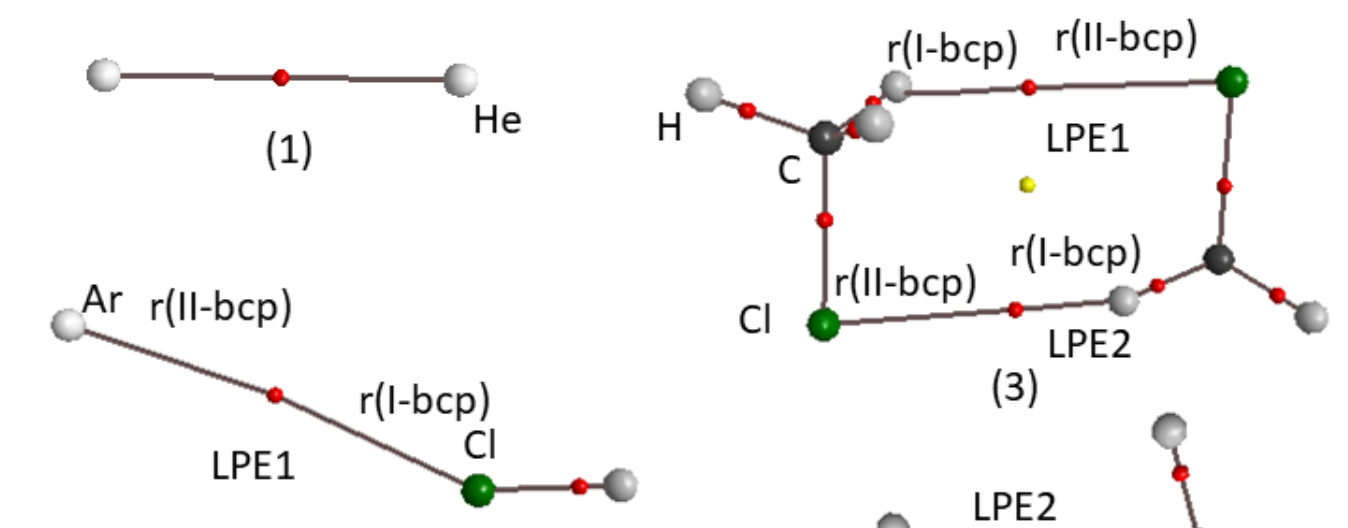

(2)

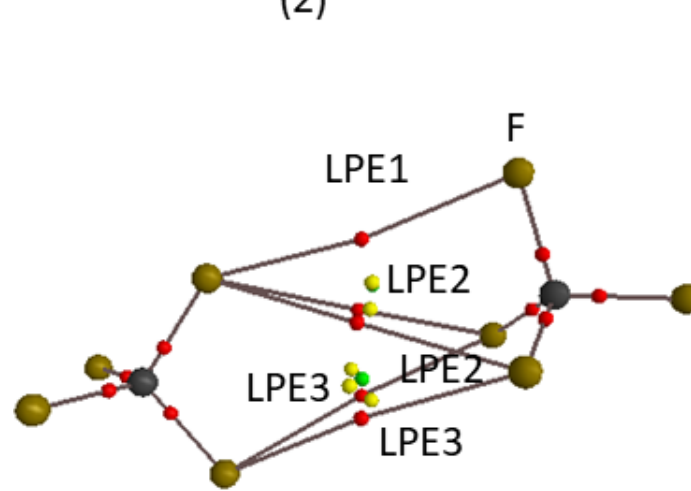

(4)

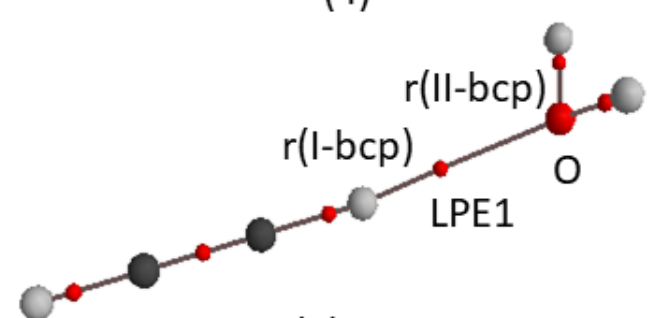

(6)

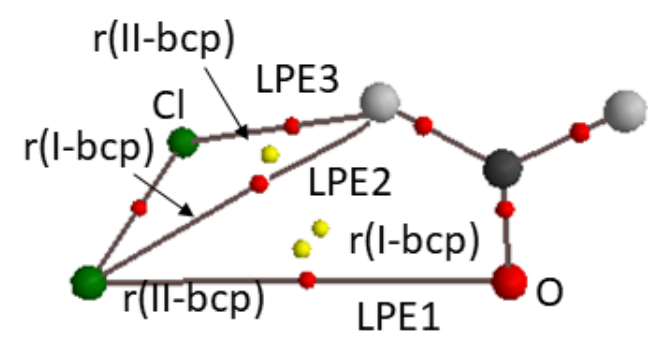

(8)
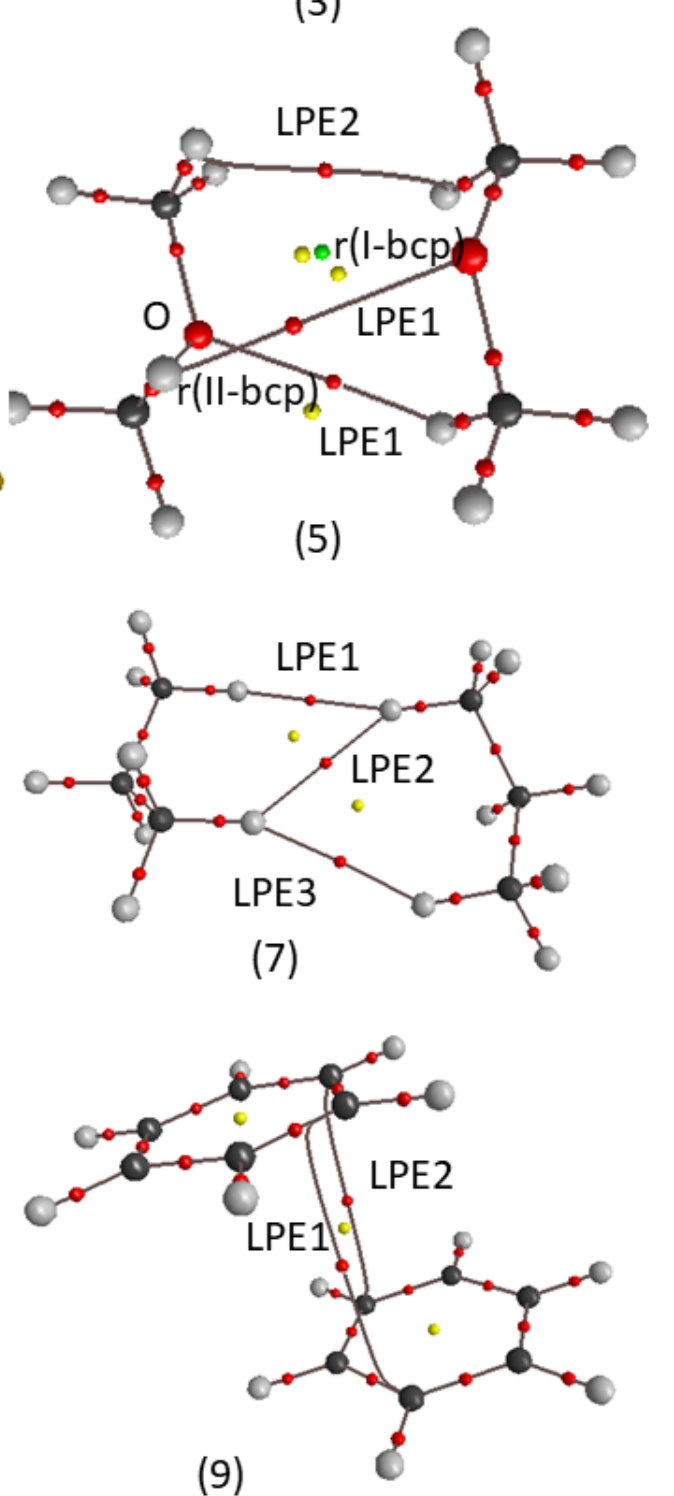

(9) 
Figure 1: Molecular graphs of helium diatomic (1), argonium-hydrogen chloride (2), chloromethame dimer (3), tetrafluoromethane dimer (4), methyl ether dimer (5), ethyne-water( $\mathrm{CH}---\mathrm{O})$ (6), propane-dimer (7), formaldehyde-chlorine (8) and benzene-dimer (9).

The Fig.2 shows the molecular graphs of 2-nitrophenol (10), along with the optimized geometry of the conformer-transition state of 2-nitrophenol (10-TS), and the complexes 11 to 14. In the complexes 12 and 13, there is a non-conventional hydrogen bond involving the hydrogen (from the halide hydrogen) and the less-substituted vinyl carbon and the triple bond itself as acceptors, respectively. The topological difference in the acceptors with respect to the interaction with the hydrogen halide is related to the asymmetry of the alkene in 12 and the symmetry of the alkyne in 13. Symmetrical alkenes/alkynes lead to symmetrical $\pi$ complexes while asymmetrical alkenes/alkynes lead to asymmetrical $\pi$ complexes in polar addition of hydrogen halides [22] to alkenes/alkynes. The complex $\mathbf{1 3}$ has a non-nuclear attractor, NNA, which is a $(3,-3)$ critical point of the charge density without any nucleus associated with it. It is more common to observe NNA in the topology of lithium clusters [42]. The complex 11 has two equivalent pair of bond paths associated with their interatomic interactions and they have the same designation (LPE1). 

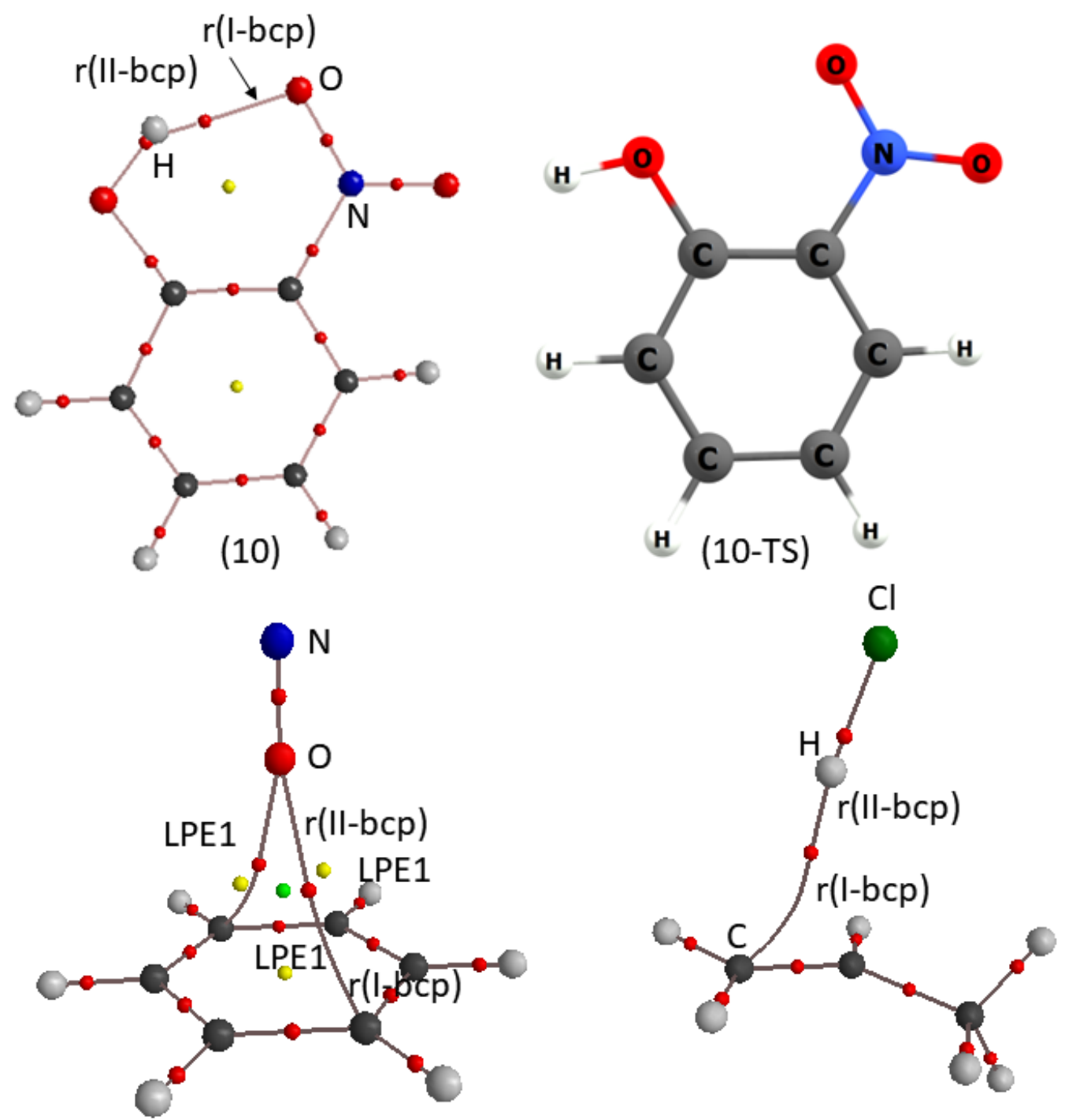

(12)

(11)

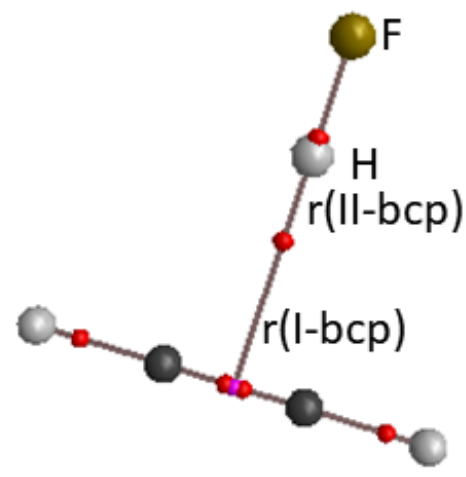

(13)

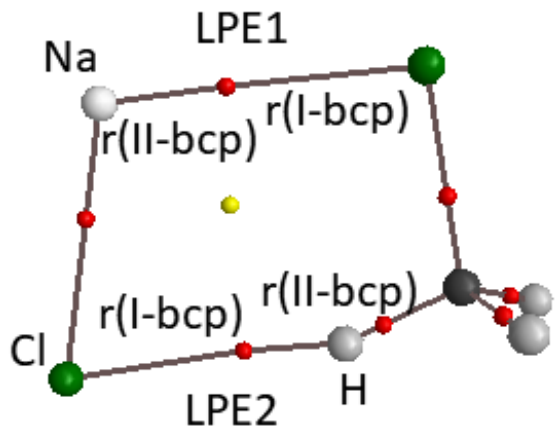

(14)

Figure 2: Molecular graphs of 2-nitrophenol (10), benzene-nitrosonium ion (11), propene-hydrogen chloride ( $\pi$ complex) (12), ethyne-hydrogen fluoride ( $\pi$ complex) (13) and chloromethane-sodium chloride (14), plus optimized geometry of the conformer-transition state of 2-nitrophenol (10-TS).

The Table 1 depicts all the topological parameters used in the local potential energy model (the Eqs. 5 and 6) to calculate the LPE for the intra/interatomic interactions in complexes/dimers from 1 to 14. Important to note that the sum of r (I-bcp) and r (II-bcp) will not give exactly the I---II interatomic distance 
because the former refers to topology and the latter to the geometry. For the dimers or complexes $\mathbf{3}, \mathbf{4}, \mathbf{5}, \mathbf{7}$, 8, 9 and 14, there are two or more interatomic interactions, i.e., two or more pair of bond paths associated with them. As a consequence, there are two or more LPE's, represented as LPE1, LP2 and LP3. The complexes 4, 5 and 11 have two equivalent pair of bond paths (two LPE1's, two LPE2's, so on) for their interatomic interactions (Figs.1 and 2). Then, the equation for the corresponding LPE in these complexes is multiplied by two. It is also important to observe that the intermolecular interaction in $\mathbf{1 3}$ (a symmetrical $\pi$ complex) involves directly the $\pi$ bond which is topologically represented by the non-nuclear attractor. Then, there is no LI associated with it and we will consider the charge density of this non-nuclear attractor as the $Z_{\text {eff. }}$

The interaction in diatomic He is so insignificant (LPE is $10^{-7}$ Hartree) that it is approximated to zero. Except for the classical hydrogen bond in $\mathbf{1 0}$ and the interaction in 11, all other interatomic interactions ranges from zero to $-2.70 \mathrm{kcal} \mathrm{mol}^{-1}$ (from LPE results), showing that these interactions (non-conventional hydrogen bond, hydrogen-hydrogen bond, $\pi$-stacking, halogen bond, ion-dipole, induced dipole-dipole interactions) are weak.

Table 1: QTAIM atomic charge, q, localization index, LI, in au., effective atomic number, $Z_{\text {eff, of the }}$ interacting atoms I and II, charge density of the bond critical point, $\rho$ (bcp), of inter/intramolecular interaction, in au.; bond path length, in Bohr unit, from atom I to bcp, r (I-bcp), and from atom II to bcp, r (II-bcp); local potential energy, LPE, in Hartree and $\mathrm{kcal} \mathrm{mol}^{-1}$ units of all studied dimers and complexes (entry 1 to 14$)$.

\begin{tabular}{|c|c|c|c|c|c|c|c|c|c|c|c|}
\hline Entry & $\begin{array}{l}\mathrm{q}(\mathrm{I}) \\
/ \mathrm{au} .\end{array}$ & $\begin{array}{l}\text { LI (I) } \\
\text { / au. }\end{array}$ & $\begin{array}{l}\text { Zeff(I) } \\
\text { / au. }\end{array}$ & $\begin{array}{l}\mathrm{q}(\mathrm{II}) \\
/ \mathrm{au} .\end{array}$ & $\begin{array}{l}\text { LI (II) } \\
/ \text { au. }\end{array}$ & $\begin{array}{c}\text { Zeff(II) } \\
\text { / au. }\end{array}$ & $\begin{array}{l}\rho(\mathrm{bcp}) \\
\text { / au. }\end{array}$ & $\begin{array}{l}\text { r (I-bcp) } \\
\text { / Bohr }\end{array}$ & $\begin{array}{l}\mathrm{r} \\
\text { (II-bcp) } \\
\text { / Bohr }\end{array}$ & $\begin{array}{c}\text { LPE / } \\
\text { Hartree }\end{array}$ & $\begin{array}{l}\text { LPE / } \\
\mathrm{kcal}^{-1} \\
\mathrm{~mol}^{-1}\end{array}$ \\
\hline 1 & -0.0006 & 1.9997 & 0.0009 & -0.0006 & 1.99970 & 0.0009 & -0.0005 & 3.0697 & 3.0697 & 0.0000 & 0.00 \\
\hline 2 & -0.2754 & 16.76 & & -0.0074 & 17.9760 & & & & & & -0.06 \\
\hline $3^{\mathrm{a}}$ & 0.0777 & 0.38 & 5418 & -0.2813 & 16.6050 & 5763 & -0.0038 & & 3.6902 & -0.0007 & -0.47 \\
\hline $3^{b}$ & 0737 & 0.3760 & 0.5503 & -0.2808 & 16.5964 & 0.6844 & -0.0066 & 2.0586 & 3.4423 & -0.0015 & -0.97 \\
\hline $4^{\mathrm{a}}$ & & & & -0.6763 & & & & & & & -0.25 \\
\hline $4^{b}$ & 6703 & 9.1 & 5657 & -0.6907 & & 61 & -0.0023 & & & 08 & -0.51 \\
\hline $4^{c}$ & .6726 & 9.1148 & 0.5578 & -0.6907 & 446 & & -0.0019 & & 3.2151 & -0.0007 & -0.41 \\
\hline $5^{a}$ & & & & & & & & & 2.2390 & 38 & -2.40 \\
\hline $5^{b}$ & & & & 067 & & & & & 2.7740 & & -0.68 \\
\hline 6 & 0.2131 & 0.2 & & -1.1677 & & & & & 2.5400 & & -2.70 \\
\hline $7^{\mathrm{a}}$ & & & & -0.01 & & & & & & & -0.77 \\
\hline $7^{b}$ & & & & & & & & & & & -0.63 \\
\hline $7^{c}$ & -0.0141 & & & -0.012 & & & & & & & -0.77 \\
\hline $8^{a}$ & -1.2115 & & $0.85^{\circ}$ & 0.0066 & 16.2 & & & & & & -0.43 \\
\hline $8^{b}$ & & 16.2 & & & & & & & & & -0.49 \\
\hline $8^{c}$ & 0.0331 & 0.4235 & 0.5434 & 0.0021 & 16.2601 & 0.7378 & -0.0036 & 2.4819 & 3.6435 & -0.0008 & -0.48 \\
\hline $9^{a}$ & -0.0047 & 3.9354 & 2.0693 & & & & & & 4.0013 & 26 & -1.64 \\
\hline $9^{b}$ & 0.0627 & & 2.0633 & & & & & & 3.3434 & -0 . & -1.89 \\
\hline 10 & & & & & & & & & & & -11.71 \\
\hline 11 & 0.0210 & 3.9248 & 2.0542 & -0.4687 & & & & & 2.4600 & -0.0205 & -12.89 \\
\hline 12 & -0.0356 & 4.0321 & 0.0035 & & 866 & 0.5026 & & & 1.6640 & -0.0027 & -1.67 \\
\hline 13 & -----(d) & -----(d) & & & & & & & & & -2.16 \\
\hline $14^{\mathrm{a}}$ & -0.3397 & 6.6595 & 0.6802 & & & & & & 2.14 & -0.0 & -0.98 \\
\hline $14^{\mathrm{b}}$ & -0.8849 & 17.6943 & 0.1906 & 0.1469 & 0.3094 & 0.5437 & -0.0114 & 3.1357 & 1.7071 & -0.0022 & -1.36 \\
\hline
\end{tabular}

a. LPE1 (see Fig.1 or Fig.2)

b. LPE2 (see Fig.1 or Fig.2)

c. LPE3 (see Fig.1) 
The Table 2 shows the total inter/intramolecular binding energy from LPE and supramolecular binding (electronic) energy, SME, methods according to the decreasing order of binding energy of the complexes 1 to 14, divided into two subsets (first, from 1 to 10, and second, from 11 to $\mathbf{1 4}$ ). In the first subset, there is a high agreement of the binding energy values between both methods LPE and SME (i.e., there is a very small energy difference between both methods for each case). In the second subset (11 to 14), there is a discrepancy of the binding energy values between both methods, mainly involving the complexes 11 and 14 (where binding energy difference is 9.77 and $15.03 \mathrm{kcal} \mathrm{mol}^{-1}$, for $\mathbf{1 4}$ and $\mathbf{1 1}$, respectively).

The decreasing order of the binding energy is equivalent for both methods in both subsets, except for the reversed order for the pairs tetrafluoromethane-dimer/formaldehyde-chlorine and benzene-dimer/methyl ether-dimer. In the first subset, the decreasing order is: $(\mathbf{1})>(\mathbf{2})>(\mathbf{4})>(\mathbf{8})>(\mathbf{3})>(\mathbf{7})>(\mathbf{6})>(\mathbf{5})>(\mathbf{9})>(\mathbf{1 0})$ for $\mathrm{LPE}$ and $(\mathbf{1})>(\mathbf{2})>(\mathbf{8})>($ 4) $>(3)>(7)>(6)>(9)>(5)>(10)$ for SME.

Table 2: Inter/intramolecular binding energy from supramolecular binding (electronic) energy, SME, and local potential energy, LPE, in $\mathrm{kcal} \mathrm{mol}^{-1}$ according to the decreasing order of the binding energy for the systems 1 to 14.

\begin{tabular}{|c|c|c|c|}
\hline Complex/dimer & $\begin{array}{c}\text { LPE } \\
/ \mathrm{kcal} \mathrm{mol}^{-1}\end{array}$ & Complex/dimer & $\begin{array}{c}\text { SME } \\
/ \mathrm{kcal} \mathrm{mol}^{-1}\end{array}$ \\
\hline Helium-diatomic (1) & 0.00 & Helium-diatomic (1) & 0.00 \\
\hline Ar-hydrogen chloride (2) & -0.06 & Ar-hydrogen chloride (2) & -0.19 \\
\hline Tetrafluoromethane-dimer (4) & -1.17 & Formaldehyde-chlorine $(\mathbf{8})$ & -0.82 \\
\hline Formaldehyde-chlorine (8) & -1.40 & Tetrafluoromethane-dimer (4) & -0.88 \\
\hline Chloromethane-dimer (3) & -1.44 & Chloromethane-dimer (3) & -1.32 \\
\hline Propane-dimer (7) & -2.17 & Propane-dimer (7) & -1.38 \\
\hline Ethyne-water $(\mathbf{6})$ & -2.70 & Ethyne-water (6) & -2.82 \\
\hline Methyl ether-dimer (5) & -3.08 & Benzene-dimer $(\mathbf{9})$ & -3.45 \\
\hline Benzene-dimer (9) & -3.53 & Methyl ether-dimer (5) & -3.51 \\
\hline 2-nitrophenol (10) & -11.71 & 2-nitrophenol (10) & -10.48 \\
\hline Propene-hydrogen chloride (12) & -1.67 & Propene-hydrogen chloride (12) & -4.33 \\
\hline Ethyne-hydrogen fluoride (13) & -2.16 & Ethyne-hydrogen fluoride (13) & -4.83 \\
\hline Chloromethane- $\mathrm{NaCl}(\mathbf{1 4})$ & -2.34 & Chloromethane- $\mathrm{NaCl}(\mathbf{1 4})$ & -12.11 \\
\hline Benzene-nitrosonium ion (11) & -12.89 & Benzene-nitrosonium ion (11) & -27.92 \\
\hline
\end{tabular}

In Fig.3(A), there is a plot of LPE vs SME for the whole set of studied complexes or dimers. The linearity is low (70.51\%). This is due to the subset of complexes from $\mathbf{1 1}$ to $\mathbf{1 4}$. The points corresponding to these complexes in Fig.3(A) appear below the trendline. When removing the data points related to 11 to 14, the coefficient of determination becomes close to $1\left(\mathrm{R}^{2}=0.9844\right)$, as depicted in Fig.3(B). Then, besides the discrepancy of the binding energy between SME and LPE for the subset of 11 to 14 (Table 3), this subset removes the linearity between SME and LPE. 


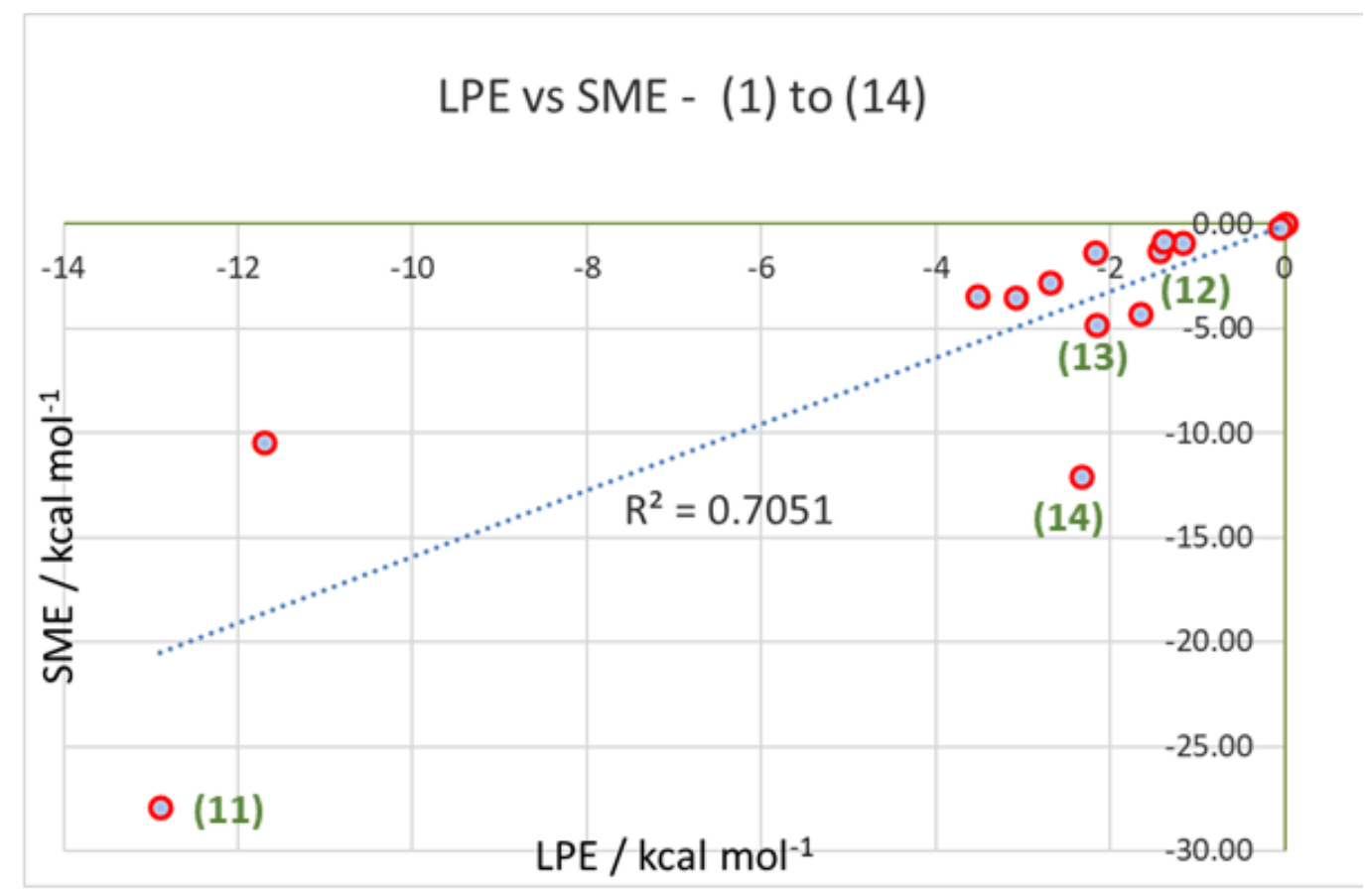

(A)

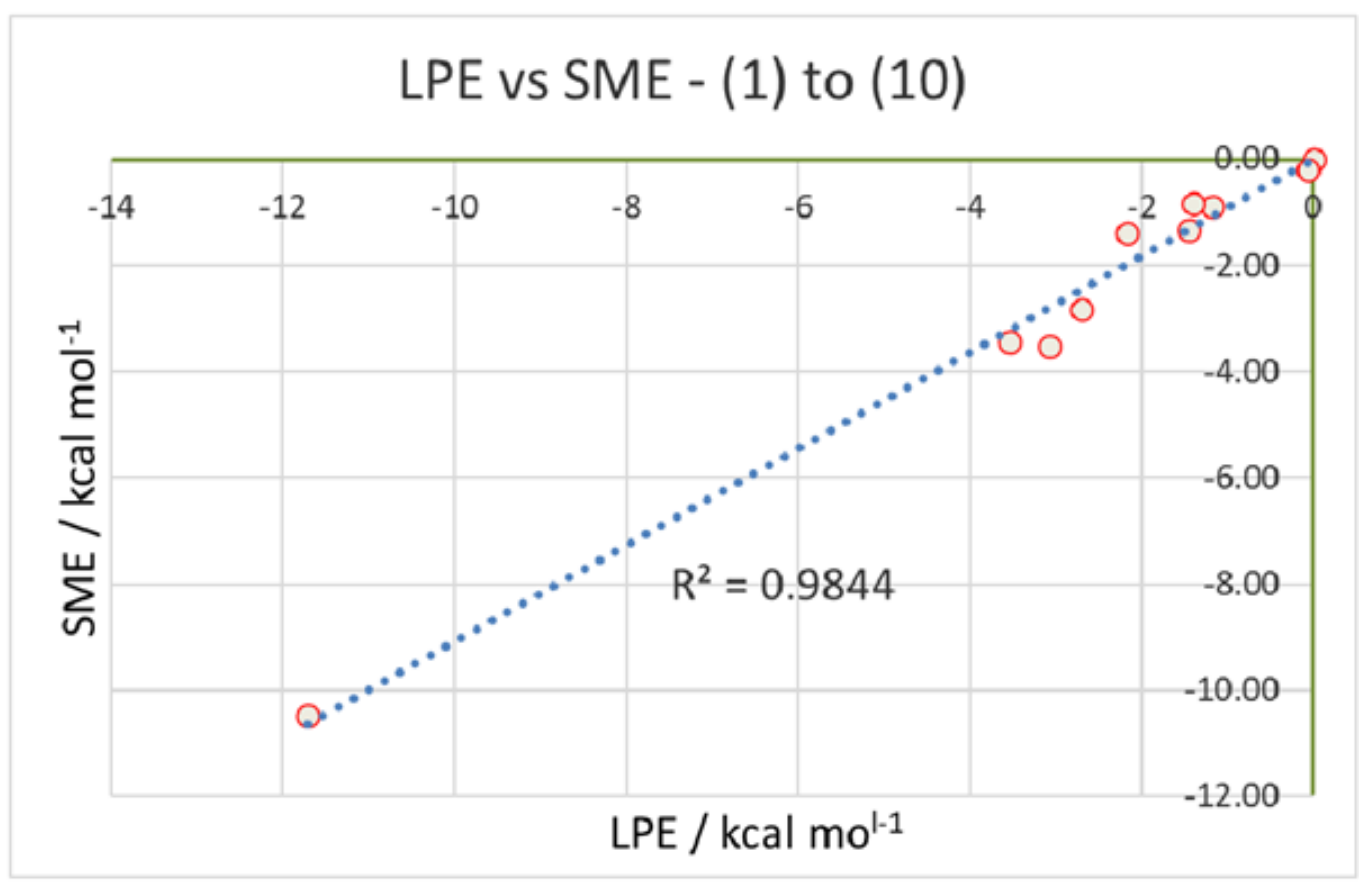

(B)

Figure 3: Plot of linear relation between local potential energy, LPE, and supramolecular binding energy, SME for: (A) for all studied complexes and dimers 1 to 14; and (B) systems 1 to $\mathbf{1 0 .}$

The Table 4 is also divided in two subsets (1 to $\mathbf{1 0}$ and $\mathbf{1 1}$ to $\mathbf{1 4}$ ) for the square difference of intra/intermolecular binding energy between LPE and SME, the corresponding average and root mean square deviation, RMSD. In the first subset, there are very small square difference values for each complex (even the higher one in $\mathbf{1 0}$ is a relatively low value). Then, according to the corresponding RMSD (0.53 kcal $\mathrm{mol}^{-1}$ ), the LPE has a high precision for this subset which is much smaller than that found between SME and LPE in the binding energy of the classical hydrogen bond in the previous work whose RMSD was three-fold 
higher [27]. This shows a high agreement between SME and LPE for inter/intramolecular interactions of nearly all kinds. On the other hand, in the second subset, the square difference of the complexes 11 to 14 is much greater, mainly those from complexes $\mathbf{1 1}$ and 14. The accumulated RMSD increases from 0.53 to 4.92 $\mathrm{kcal} \mathrm{mol}^{-1}$, nine-fold higher than that from the first subset or nine-fold less precise.

As to the second subset (from $\mathbf{1 1}$ to $\mathbf{1 4}$ ), it is important to add that the complexes $\mathbf{1 2}$ and $\mathbf{1 3}$ do have only a moderate discrepancy between LPE and SME (having average binding energy difference around 2.7 $\mathrm{kcal} \mathrm{mol}^{-1}$ ) which is comparable to some binding energy differences between SME and LPE in the previous work involving classical hydrogen bond [27]. Although the discrepancies from complexes 12 and $\mathbf{1 3}$ is relatively low, the SME-LPE data of the complexes $\mathbf{1 2}$ and $\mathbf{1 3}$ decrease the linearity between SME and LPE from $98.44 \%$ (from the first subset $\mathbf{1}$ to $\mathbf{1 0}$ ) to $83.31 \%$ (involving $\mathbf{1}$ to $\mathbf{1 0}$, plus $\mathbf{1 2}$ and $\mathbf{1 3}$ ).

Table 4: Square difference between reference variable and dependent variable of inter/intramolecular binding energy from SME and LPE, $\left(\mathrm{LPE}_{\mathrm{i}}-\mathrm{SME}_{\mathrm{i}}\right)^{2}$ from dimers and complexes $\mathbf{1}$ to $\mathbf{1 0}$ along with their corresponding average values and root mean square deviation values, RMSD, in $\mathrm{kcal} \mathrm{mol}^{-1}$, plus corresponding values for $\left(\mathrm{LPE}_{\mathrm{i}}-\mathrm{SME}_{\mathrm{i}}\right)^{2}$ from dimers and complexes 11 to $\mathbf{1 4}$ along with their corresponding accumulated average values and root mean square deviation values, RMSD, in $\mathrm{kcal} \mathrm{mol}^{-1}$.

\begin{tabular}{llr}
\hline Entry & System & $\left(\mathrm{LPE}_{\mathrm{i}}-\mathrm{SME}_{\mathrm{i}}\right)^{2}$ \\
\hline 1 & helium-diatomic & 0.00 \\
2 & Ar-hydrogen chloride & 0.02 \\
3 & chloromethane-dimer & 0.01 \\
4 & tetrafluoromethane-dimer & 0.08 \\
5 & Methyl ether-dimer & 0.18 \\
6 & ethyne-water $(\mathrm{CH}---\mathrm{O})$ & 0.02 \\
7 & propane-dimer & 0.62 \\
8 & formaldehyde-chlorine & 0.34 \\
9 & benzene-dimer & 0.01 \\
10 & 2-nitrophenol & 1.51 \\
\hline & Average & $\mathbf{0 . 2 8}$ \\
\hline & RMSD/ kcal mol & $\mathbf{0 . 5 3}$ \\
\hline & System & $\left(\mathrm{LPE}_{\mathrm{i}}-\mathrm{SME}_{\mathrm{i}}\right)^{2}$ \\
\hline 11 & benzene-nitrosonium ion & 225.90 \\
12 & propene-hydrogen chloride $(\pi)$ & 7.10 \\
13 & ethyne-hydrogen fluoride $(\pi)$ & 7.13 \\
14 & chloromethane-NaCl & 95.45 \\
\hline & Accumulated average & $\mathbf{2 4 . 1 7}$ \\
\hline & Accumulated RMSD \\
\hline
\end{tabular}

a. Average value of $\left(\mathrm{LPE}_{\mathrm{i}}-\mathrm{SME}_{\mathrm{i}}\right)^{2}$ from 1 to 14.

b. RMSD value from 1 to 14 .

The Fig.4(A) shows the energy decomposition analysis, EDA, of the binding energy from SAPT-DFT method for the complexes or dimers 3 to 14 and Fig.4(B) displays the EDA of the LMOEDA method for the complexes or dimers 3 to 14., excluding the complex 11 which could not be calculated in this method. We can see in both figures that in the subset from $\mathbf{1}$ to $\mathbf{1 0}$, the electrostatic and/or the dispersion component(s) are predominant attractive contributions to the total binding energy. Then, electrostatic and dispersion components are important for the precision of the LPE model. In the subset of complexes $\mathbf{1 1}$ to 14, the polarization (from LMOEDA) or induction (from SAPT-DFT) component has a higher contribution when 
comparing to that from $\mathbf{3}$ to 10. Then, we can assume that complexes or dimers with high polarization or induction component from EDA leads to the discrepancy between SME and LPE.

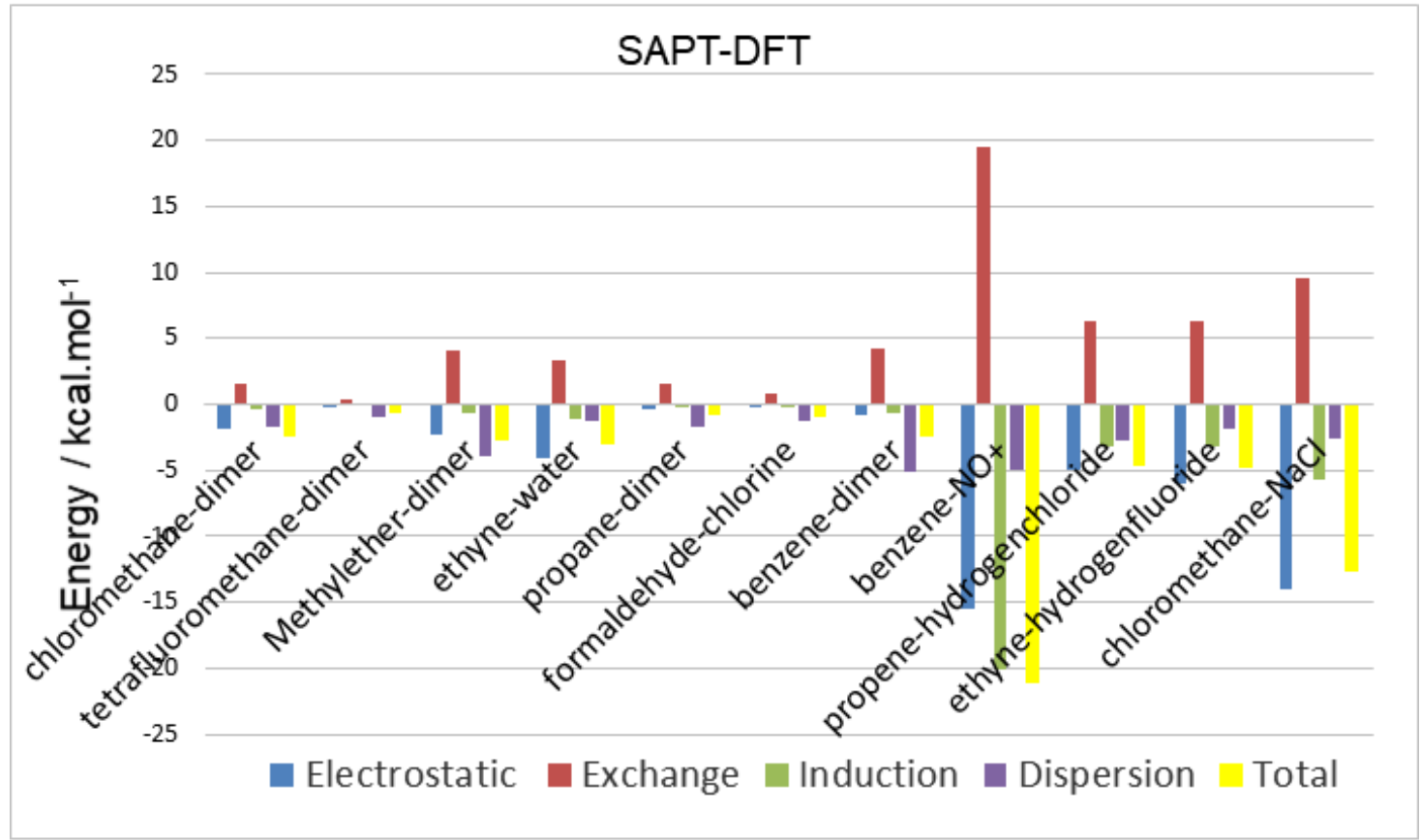

(A)

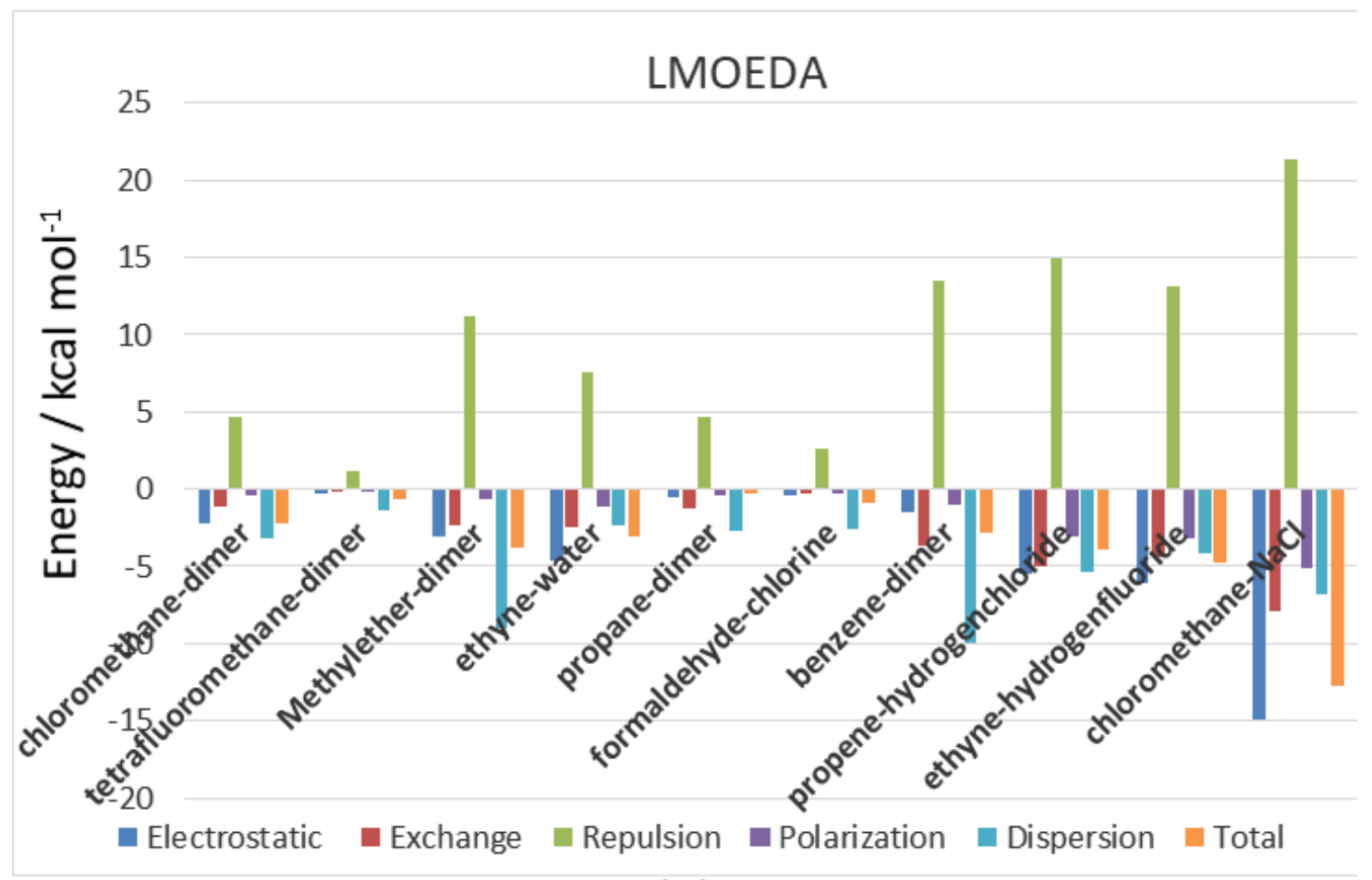

(B)

Figure 4: Energy decomposition analysis of dimers and complexes from 3 to 14 using: (A) LMOEDA, excluding complex 11, and (B) SAPT-DFT. 
This work extends the analysis of the local potential energy, LPE, as a tool to quantify the inter/intramolecular binding energy from classical hydrogen bond in our previous work [27] to several other interatomic interactions (non-conventional hydrogen bonds, $\pi$-stacking, halogen bond, hydrogen-hydrogen bond, induced dipole-dipole interaction, ion-dipole interaction, including classical hydrogen bond with formal partial charge in the acceptor), using supramolecular binding energy, SME, as a reference.

We found that LPE can be successfully applied to a wide range of inter/intramolecular interactions with a precision three-fold higher than that found for classical hydrogen bond [27]. There is a very high linearity between SME and LPE (98.44\%) and a very low RMSD $\left(0.53 \mathrm{kcal} \mathrm{mol}^{-1}\right)$ for the subset of complexes/dimers from 1 to 10. Even complexes $\mathbf{1 2}$ and $\mathbf{1 3}$ do have only a moderate discrepancy (around $2.70 \mathrm{kcal} \mathrm{mol}^{-1}$ ) between SME and LPE, although they decrease the linearity to $83.31 \%$. When considering the whole second subset (from 11 to 14 ), the linearity decreases to $70.51 \%$ and the precision of LPE decreases nine-fold. The highest discrepancy is found in complexes $\mathbf{1 1}$ and $\mathbf{1 4}$ (where binding energy difference between SME and LPE is 9.77 and $15.03 \mathrm{kcal} \mathrm{mol}^{-1}$, respectively). Since these complexes have ions interacting with neutral molecules, we can see that the LPE cannot be used for ions interacting with $\pi$-bonded systems or polar molecules.

From the energy decomposition analysis using LMOEDA and SAPT-DFT, we found that the subset from $\mathbf{1 1}$ to $\mathbf{1 4}$ has an important contribution of the polarization (or induction) component, which implies that the polarization component decreases the precision of LPE. On the other hand, for the first subset, both electrostatic and dispersion components are predominant indicating that these components increase the precision of the LPE model.

\section{REFERENCES}

[1] P.A. Kollman, L.C. Allen, The Theory of the Hydrogen Bond, Chem. Commun. 72 (1972) 283-303.

[2] A.S.N. Murthy, C.N.R. Rao, Recent Theoretical Studies of the Hydrogen Bond, J. Mol. Struct. 6 (1970) 253-282.

[3] B.J. Emsley, Very Strong Hydrogen Bonding, Chem. Soc. Rev. 9 (1980) 91-124.

[4] C.L. Perrin, J.B. Nielson, "Strong” Hydrogen Bonds in Chemistry and Biology, Annu. Rev. Phys. Chem. 48 (1997) 511-544.

[5] E.N. Baker, R.E. Hubbard, Hydrogen Bonding In Globular Proteins, Prog. Biophys. Molec. Biol. 44 (1984) 97-179.

[6] A.R. Fersht, The hydrogen bond in molecular recognition, Trends Biochem. Sci. 12 (1987) 301-304.

[7] S.E. Harding, G. Channell, M.K. Phillips-Jones, The discovery of hydrogen bonds in DNA and a re-evaluation of the 1948 Creeth two-chain model for its structure, Biochem. Soc. Trans. 46 (2018) 1171-1182.

[8] L. Pauling, The Shared-Electron Chemical Bond, Proc. Natl. Acad. Sci. 14 (1928) 359 LP - 362.

[9] S.J. Grabowski, What Is the Covalency of Hydrogen Bonding ?, Chem. Rev. 111 (2011) 2597-2625.

[10] B. Jeziorski, R. Moszynski, K. Szalewicz, Perturbation Theory Approach to Intermolecular Potential Energy Surfaces of van der Waals Complexes, Chem. Rev. 94 (2002) 1887-1930.

[11] E.G. Hohenstein, C.D. Sherrill, Density fitting of intramonomer correlation effects in symmetry-adapted perturbation theory, J. Chem. Phys. 133 (2010) 014101.

[12] P. Su, H. Li, Energy decomposition analysis of covalent bonds and intermolecular interactions, J. 
Chem. Phys. 131 (2009) 14102.

[13] P. Sanghera, Quantum Physics for Scientists and Technologists: Fundamental Principles and Applications for Biologists, Chemists, Computer Scientists, and Nanotechnologists, 1st editio, WIley-Interscience, Hoboken, 2011.

[14] S.J. Grabowski, An estimation of strength of intramolecular hydrogen bonds - ab initio and AIM studies, J. Mol. Struct. 562 (2001) 137-143.

[15] E. Espinosa, E. Molins, C. Lecomte, Hydrogen bond strengths revealed by topological analyses of experimentally observed electron densities, Chem. Phys. Lett. 285 (1998) 170-173.

[16] S.J. Grabowski, A new measure of hydrogen bonding strength - ab initio and atoms in molecules studies, Chem. Phys. Lett. 338 (2001) 361-366.

[17] S.J. Grabowski, Ab Initio Calculations on Conventional and Unconventional Hydrogen BondsStudy of the Hydrogen Bond Strength, J. Phys. Chem. A. 105 (2001) 10739-10746.

[18] S.J. Grabowski, W.A. Sokalski, E. Dyguda, J. Leszczyński, Quantitative Classification of Covalent and Noncovalent H-Bonds, J. Phys. Chem. B. 110 (2006) 6444-6446.

[19] J. N. Woodford, Density Functional Theory and Atoms-in-Molecules Investigation of Intramolecular Hydrogen Bonding in Derivatives of Malonaldehyde and Implications for Resonance-Assisted Hydrogen Bonding, J. Phys. Chem. A. 111 (2007) 8519-8530.

[20] I. Alkorta, I. Rozas, J. Elguero, Non-conventional hydrogen bonds, Chem. Soc. Rev. 27 (1998) 163170.

[21] C.L. Firme, Revisiting the mechanism for the polar hydrochlorination of alkenes, J. Mol. Model. 25 (2019).

[22] C.L. Firme, Introductory Organic Chemistry and Hydrocarbons - a Physical Chemistry Approach, 1st ed., CRC Press, Boca Raton, 2020.

[23] C.L. Firme, D.M. Araújo, Revisiting electronic nature and geometric parameters of cyclophanes and their relation with stability - DFT, QTAIM and NCI study, Comput. Theor. Chem. 1135 (2018).

[24] C.L. Firme, Deeper Insights into Conformational Analysis of cis -Butene and 1-Alkenes as Monomers and Dimers: QTAIM, NCI, and DFT Approach , J. Chem. (2019).

[25] N.K.V. Monteiro, C.L. Firme, Hydrogen-hydrogen bonds in highly branched alkanes and in alkane complexes: A DFT, ab initio, QTAIM, and ELF study, J. Phys. Chem. A. 118 (2014).

[26] G. Cavallo, P. Metrangolo, R. Milani, T. Pilati, A. Priimagi, G. Resnati, G. Terraneo, The Halogen Bond, Chem. Rev. 116 (2016) 2478-2601.

[27] C.L. Firme, Local potential energy: a novel QTAIM tool to quantify the binding energy of classical hydrogen bonds, Chem. Phys. Lett. (2020) 137593.

[28] H.B. Schlegel, Optimization of equilibrium geometries and transition structures, J. Comput. Chem. 3 (1982) 214-218.

[29] P. Pulay, Improved SCF convergence acceleration, J. Comput. Chem. 3 (1982) 556-560.

[30] X. Li, M.J. Frisch, Energy-Represented Direct Inversion in the Iterative Subspace within a Hybrid Geometry Optimization Method, J. Chem. Theory Comput. 2 (2006) 835-839.

[31] M.J. Frisch, G.W. Trucks, H.B. Schlegel, et al., Gaussian 09. Revision A.01, (2009).

[32] J. Da Chai, M. Head-Gordon, Long-range corrected hybrid density functionals with damped atom-atom dispersion corrections, Phys. Chem. Chem. Phys. 10 (2008) 6615-6620.

[33] T.H. Dunning, Gaussian basis sets for use in correlated molecular calculations. I. The atoms boron through neon and hydrogen, J. Chem. Phys. 90 (1989) 1007-1023. 
[34] R.A. Kendall, T.H. Dunning, R.J. Harrison, Electron affinities of the first-row atoms revisited. Systematic basis sets and wave functions, J. Chem. Phys. 96 (1992) 6796-6806.

[35] K.S. Thanthiriwatte, E.G. Hohenstein, L.A. Burns, C.D. Sherrill, Assessment of the Performance of DFT and DFT-D Methods for Describing Distance Dependence of Hydrogen-Bonded Interactions, J. Chem. Theory Comput. 7 (2011) 88-96.

[36] J. M. Turney, A. C. Simmonett, R. M. Parrish,et al., Psi4: An open-source ab initio electronic structure program, WIREs Comput. Mol. Sci. 2 (2012) 556-565.

[37] M. W. Schmidt, K. K. Baldridge, J. A. Boatz, et al., General atomic and molecular electronic structure system, J. Comput. Chem. 14 (1993) 1347-1363.

[38] Y. Zhao, D.G. Truhlar, The M06 suite of density functionals for main group thermochemistry, thermochemical kinetics, noncovalent interactions, excited states, and transition elements: two new functionals and systematic testing of four M06-class functionals and 12 other function, Theor. Chem. Acc. 120 (2008) 215-241.

[39] F. Biegler-Konig, J. Schonbohm, D. Bayles, Software news and updates - AIM2000 - A program to analyze and visualize atoms in molecules, J. Comput. Chem. 22 (2001) 545-559.

[40] Y.G. Wang, N.H. Werstiuk, A practical and efficient method to calculate AIM localization and delocalization indices at post-HF levels of theory, J. Comput. Chem. 24 (2003) 379-385.

[41] G.R. Desiraju, The C-H $\cdots$ O Hydrogen Bond: Structural Implications and Supramolecular Design, Acc. Chem. Res. 29 (1996) 441-449.

[42] C. Gatti, P. Fantucci, G. Pacchioni, Charge density topological study of bonding in lithium clusters, Theor. Chim. Acta. 72 (1987) 433-458. 